The Geneva Papers on Risk and Insurance, 18 (No. 69, October 1993), 399-402

\title{
La Bancassurance, à la fortune du mot
}

\author{
par Jean Peyrelevade*
}

A qui doute de la vitalité de la langue française, la bancassurance apporte un heureux démenti. Irrécusable par l'Académie ou la très officielle Commission de Terminologie du Ministère des Finances, le mot s'est imposé à tous, avant, suprême hommage, de nous être empruntés par nos amis britanniques, sans vergogne ni procès.

Mais le mot a si bien fait mouche qu'il a occulté la chose, et a recouvert des acceptations de plus en plus diverses. Bancassurance, la création de compagnies d'assurance Vie puis d'assurance dommages par des banques qui distribuent leurs contrats aux guichets. Bancassurance, les fusions qui ont donné naissance à de vastes conglomérats financiers, aux Pays-Bas (Internationale Nederlanden) et en France (GAN - C.I.C.). Bancassurance encore, la distribution de produits financiers par des réseaux commerciaux d'assurance, même si je dois à la vérité de mentionner le nom «d'assurfinance» que mes concurrents préfèrent employer, quoique la facture en soit moins sûre.

Au-delà de la sémantique qu'on vend dans les séminaires de consultants, mon propos veut revenir sur la logique économique de ces opérations, montrant qu'il existe bien un fil directeur qui les unit et que la «bancassurance» est le fruit, paradoxal mais inévitable, d'une alliance et d'une concurrence également renforcées entre les deux métiers.

Arrêtons-nous un instant sur le passé proche. Comme la banque et l'assurance étaient jolies, quand chacune restant chez elle, assurés et déposants étaient bien gardés. Au banquier, l'octroi des crédits et la tenue des comptes des clients; le risque n'est pris qu'à l'actif du bilan. A l'assureur, la garantie donnée contre les aléas de la vie économique ou de la vie tout court; le risque est pris essentiellement au passif du bilan, le moins possible à l'actif. Entre les deux, l'épargne se répartit selon sa nature; purement financière, elle va vers la banque; recherchant des garanties annexes, elle recourt à la technique de l'assureur. Dire que la concurrence entre les métiers n'avait pas cours serait trop dire; mais ces deux mondes, bien typés sociologiquement et culturellement, pouvaient s'ignorer sans tourment.

Ce confort a pris fin. Banquiers et assureurs ont commencé de retirer une moindre rentabilité de leurs activités les plus spécifiques - crédit et gestion des comptes pour les uns, assurance dommages pour les autres - et se sont affrontés de plus en plus ouvertement pour

\footnotetext{
* Président, Union des Assurances de Paris.
} 
le marché le plus prometteur: l'épargne des ménages. Voulant également appréhender les flux d'épargne, les uns et les autres ont été amenés à cerner de mieux en mieux les besoins globaux du client, à développer une analyse dite de «client complet» qui s'oppose à l'analyse traditionnelle par type de produits et qui conduit inéluctablement chacun à souhaiter pouvoir proposer les produits de l'autre.

L'exemple le plus achevé de cctte démarche, nous l'observons en France chez le Crédit Agricole, qui vcut offrir à ses clients l'ensemble des produits bancaires et d'assurance, en Vie (Prédica) comme en Dommages (Pacifica) : dans les zones rurales où le Crédit Agricole domine de façon écrasante le paysage bancaire, le client sera entièrement pris en charge, sans avoir l'occasion de rencontrer un autre interlocuteur financier. Si les investissements initiaux sont lourds, le succès commercial semble devoir êtrc au rendez-vous.

La concurrence «fratricide» des banquiers et des assureurs se développe d'autant plus facilement que lcs frontières entre les métiers tendent à s'estomper. La bataille porte désormais aussi bien sur les produits que sur les réseaux et enfin, chose moins souvent relevée, que sur la matière première des financiers: les fonds propres.

Les produits d'abord. Ils fondaient l'identité et protégeaient le pré carré de chacun. Or rien n'a empêché les banquiers, lorsqu'il est devenu évident que l'assurance Vie offrait en France la forme de placement à long terme la plus avantageuse, de créer leurs propres filiales et de prendre une part croissante de ce marché, ou plutôt de reprendre leur partie de l'épargne qu'ils avaient laissée finir chez les assureurs. Les assureurs sont venus proposer à leurs clients les produits bancaires et financiers de plus court terme qui manquaient à leur panoplie, que ces produits soient fabriqués sous leur propre marque ou sous celle de banques filiales ou associées. Les banquiers prennent pied dans le domaine de l'assurance dommages, pour couvrir les besoins de base des familles, avec des contrats automobiles, habitation ou santé ; le Crédit Mutuel avait ouvert la voie, il y a déjà 20 ans, sur un marché régional; aujourd'hui le Crédit Agricole, la B.N.P., le C.I.C., le Crédit Lyonnais le rejoignent avec de grandes ambitions. Les assureurs distribuent dans leurs réseaux des crédits, qui fidélisent la clientèle et permettent des montages patrimoniaux intéressants quand ils sont judicieusement associés à l'assurance Vie, etc...

Je ne doute pas que, la concurrence s'avivant et l'ingéniosité des financiers aidant, la liste se prolonge encore longtemps. S'il serait dangereux de sous-estimer le savoir faire spécifique à chaque métier, et particulièrement à l'analyse du crédit chez le banquier, à la gestion des sinistres chez l'assureur, il serait tout aussi dangereux de sous-estimer la capacité d'une institution financière déterminée, puissante et maîtresse d'une base de clientèle à pénétrer sur un marché connexe au sien.

Les réseaux commerciaux sont au cœur de notre débat. C'est aussi là que l'on observe les différences les plus marquées entre banque et assurance. Les réseaux commerciaux d'assureurs traditionnels français comme l'U.A.P., si j'écarte le courtage qui ne constitue pas à proprement à parler un réseau, se répartissent entre des agents généraux, professionnels indépendants mais représentants exclusifs d'une compagnie, à forte dominante dommages, et de salariés commissionnés, dont la chute prépondérante est l'assurance Vie. Les banquiers ont, à l'opposé, une maîtrise directe de leurs réseaux d'agences.

Les banquiers ont aujourd'hui la partie belle dans cette concurrence. Usant, parfois abusant, de la force que leur confère leur relation de comptc avec leur client, ils sont particulièrement bien placés pour détecter et satisfaire ses besoins. Les réseaux d'assureurs 
n'ont pas cet avantage. Enfin les banquiers pratiquent des chargements faibles, qui leur donnent une bonne position en compétitivité-prix.

Je crois, et à l'U.A.P. nous avons beaucoup æuvré dans ce sens, que ce déséquilibre devrait à terme sc corriger. Les chargements se rapprocheront. D'un côté les assureurs commencent de tirer les avantages de leur effort accru de compétitivité, grâce à la réduction des frais généraux, à l'accroissement de la productivité et à la modernisation des structures de rémuneration. La marge d'intermédiation de I'U.A.P. Vie est ainsi descendue de 2 points en 4 ans, passant à 3,2\% fin 1992. De l'autre les banquiers, soumis à une pression concurrentielle avivée, seront amenés à mieux tarifer le coût de distribution de leurs réseaux, actuellcment noyé dans une comptabilité analytique qui, sauf exception, ne fonctionne pas par lignes de produits. Par ailleurs, les réseaux salariés d'assurance évoluent vers un rôle de conseiller global en patrimoine, avec des moyens (diagnostic informatique) qui les rendront au moins aussi performants que les banquiers.

Le seul avantage décisif que retiendra le banquicr scra la relation de compte avec le client et l'influence qu'elle procurc. Encore ce point lui-même commence-t-il à évoluer avec le développement, en direction des clients les plus aisés, de la «banque sans guichet» dont Cortal en France est l'exemple le plus abouti.

Certains assureurs distribuent des comptes rémunérés de ce type, logés dans leurs filiales bancaires, qui leur permettront progressivement d'établir une telle relation. La formule, qui paraît appelée à un grand avenir, est en quelque sorte la réponse du berger à la bergère, face aux compagnies Vie des banques.

Se pose enfin la question de l'utilisation des fonds propres. Les métiers financiers, de banque comme d'assurance, ont en commun de reposer sur un effet de levier entre des fonds propres, gages de la solvabilité des entreprises, et des engagements, crédits et participations pour les uns, primes dommages et provisions mathématiques Vie pour les autres. Les pouvoirs publics limitent, réglementent et surveillent cet effet de levier, sous forme de la marge de solvabilité pour les assureurs, de ratio européen de solvabilité pour les banquiers. Il est clair que la capacité de démultiplier un franc de fonds propres est un facteur clé de compétitivité. Or la réglementation actuelle permettant aux banquiers d'investir dans l'assurance, aux assureurs d'investir dans la banque, sans défalquer de ces investissements des fonds propres utilisés dans les calculs de solvabilité, ce qui permet ce double effet de levier. Il y a donc un risque de distorsion de concurrence anormale entre des institutions qui utilisent deux fois, voire plusieurs fois, les mêmes fonds propres et d'autres qui ne recourent pas à cette commodité. Au total, banquiers et assureurs se retrouvent là encore concurrents pour obtenir le meilleur impact de leurs fonds propres sur leur activité avec un avantage pour les assureurs, historiquement mieux capitalisés.

Quelles stratégies adopter face à cette concurrence entre métiers? Elles ont été nombreuses et variables selon les marchés et les pays, mais il n'est pas sans intérêt d'en analyser quelques-uns en particulier.

Pratiquement toutes les banques ont investi pour créer et contrôler elles-mêmes en totalité une filiale d'assurance Vie. Cette approche a été systématisée par le Crédit Agricole en France, qui a, comme je l'ai dit précédemment, créé de même une filiale dommages. Elle procure l'avantage d'une maîtrise complète de la gamme de produits financiers; elle présente l'inconvénient d'être coûteuse en capitaux et le serait plus encore si le doublc effet de levier entre banques et assurances était interdit ou limité. 
Une stratégie d'intégration plus systématique encore a été poursuivie par certains pays européens, qui n'ont pas hésité à fusionner deux entités préexistantes de banque et d'assurance. Ainsi est bâtie, en théorie, une gamme complète de produits et de services à offrir aux clients de tels conglomérats financiers.

$\mathrm{J}^{\circ}$ 'dois pourtant avouer mon doute intellectuel devant ces constructions. Je crains que la mise en ceuvre pratique des synergies entre des maisons aussi importantes, avec leur passé et leur culture propre, ne soit lente et ne comporte les difficultés bien connues des opérations de fusion.

J'aimerais m'arrêter un instant sur notre propre réponse, le rapprochement entre l'U.A.P. et la B.N.P., dont la portée est parfois mal comprise. Notre alliance, fondée sur un actionnariat équilibré, chacun ayant une participation de $10 \%$ chez l'autre, permet de développer une relation d'égal à égal pour trouver entre nous une opinion économique. Notre opération commune en assurance dommages en fournit l'illustration: nous y sommes associés à égalité en termes économiques, en partageant le résultat de l'ensemble de l'activité d'assurance et de distribution.

Nous réalisons une économie considérable en ne créant pas de compagnie d'assurance ou de réseau de distribution et de gestion des sinistres ex nihilo, mais en utilisant des moyens existants: le réseau B.N.P., la compagnie d'assurance U.A.P. Dommages et le réseau des Agents Généraux U.A.P. pour la gestion des sinistres. Si le succès commerical est à la hauteur de nos espérances, l'investissement ayant été contenu, pour l'essentiel, à la formation des forces de vente, le résultat économique de l'opération sera plus qu'honorable.

Notre relation privilégiée avec la B.N.P. est ainsi appelée à se développer. Nous demeurons indépendants, et même concurrents sur une large part de la gamme des produits, tout en développant, chaque fois que cela a un sens économique, des opérations d'attaque en commun de tel ou tel marché. Nous avons en quelque sorte concrétisé le concept d'alliance compétitive qui est à la racine de la bancassurance.

Cette situation singulière, faut-il la redouter? Je ne le crois pas. Certes elle ajoute parfois à des excès concurrentiels au nom d'une transparence financière mal comprise, qui privent les institutions de banque et d'assurance d'une rémunération normale de leurs fonds propres et risquent de provoquer une crise par de l'intermédiation financière, au détriment de tout le marché. Un retour à la vérité économique s'imposera: que ce soit sur l'allocation des fonds propres, sous l'influence des autorités de Bruxelles qui réfléchissent à une réglementation du double effet de levier, ou sur la tarification réaliste des coûts de distribution et d'intermédiation. Dès lors que cet objectif est atteint, le rapprochement de la banque et de l'assurance répond à une nécessité commerciale et pourra favoriser, à plus long terme, un renouveau indispensable de la fonction de l'épargne dans nos économies.

Je conclurai par ce point. Nos économies, singulièrement l'économie française, doivent faire face à une insuffisance d'épargne. Un chèque d'un montant déraisonnable a été tiré sur les générations à venir, du fait d'un régime retraite axé sur le «tout répartition»; ce chèque est aujourd'hui sans provision. Le défi des années à venir est de bâtir un système d'intermédiation financière capable de dynamiser l'épargne et de l'orienter vers des besoins à long terme de l'économie : sur ce terrain là, banquiers et assureurs seront autant solidaires que concurrents. 